\title{
PROGRAMA DE TRATAMIENTO AGONISTA OPIÁCEOS EN UNA PRISIÓN \\ EN PUERTO RICO Y \\ NECESIDADES DE LAS \\ FAMILIAS DE LOS CONFINADOS
}

\author{
Gisela Negrón Velázquez ${ }^{1}$ \\ Amarilis Figueroa ${ }^{2}$ \\ Ángel A. González Carrasquillo ${ }^{3}$
}

\section{Resumen}

Este artículo presenta y discute el desarrollo y la pertinencia social de un Programa de Tratamiento de Agonista Opiáceos (PTAO) del Departamento de Corrección y Rehabilitación en Puerto Rico. Analiza críticamente el contexto en el cual el mismo se está creando y expone cuáles son las expectativas de organizaciones profesionales y agencias acreditadotas respecto a la integración de la familia del confinado en su proceso de recuperación de trastornos adictivos con opióides. Se plantean también, las diversas necesidades de familiares de confinados, según reportadas en la literatura, y expone la urgencia de examinar las necesidades de aquellos familiares de confinados que participan y participarán en programas de tratamiento para el abuso y dependencia de opiáceos en instituciones penales en la Isla. Para la profesión de trabajo social que responde a valores y principios éticos claros, se justifica su involucramiento en los procesos de decriminalización de las adicciones, y sea a través de programas como el PTAO para confinados y familiares, o en otros escenarios y niveles de intervención.

Descriptores: Programa de Tratamiento Agonista Opiáceos (PTAO) en prisión; Departamento de Corrección y Rehabilitación de Puerto Rico;

\footnotetext{
${ }^{1}$ Profesora en el Departamento de Trabajo Social, Facultad de Ciencias Sociales, Universidad de Puerto Rico en Río Piedras.

${ }^{2}$ Estudiante de Trabajo Social y asistente de investigación, Universidad de Puerto Rico en Río Piedras.

${ }^{3}$ Adictólogo y Consultor para el Departamento de Corrección y Rehabilitación.
} 
confinados; adicciones; necesidades de la familia; servicios farmacológicos y psico-sociales; estigma; reincidencia criminal.

\section{Abstract}

This article describes the recent development of a Prison Opiate Agonist Treatment Program (OATP) sponsor by the Department of Correction and Rehabilitation of Puerto Rico, and it also presents a critical analysis of the context in which the program is been developed. Moreover, the article discusses what are the expectations of professional organizations and various accreditation boards regarding the integration of family members in the treatment process of prisoners who are opiate dependent, and present a review of the literature about the needs of these families. The desirability to integrate the family in the treatment process of opiate dependent prisoners raised the urgent need to conduct a local need assessment among families from our program. Ethical values and principles of the social work profession justify and promote the presence of social workers in the decriminalization process of substance disorders, Still, more presence of this professional group is needed.

Key words: Opiate Agonist Treatment Program (OATP) in prison; Department of Correction and Rehabilitation of Puerto Rico, substance disorders; family needs; pharmacological and psico-social services; stigma; recidivism.

\section{Introducción}

El Programa de Tratamiento de Agonistas Opiáceos (PTAO) de PR es el único programa en prisiones en el territorio de E.E.U.U. ya que en ese país sólo los confinados que no han sido sentenciados (sumariados) o que participan en programas de desvío en algunos estados reciben tratamiento con metadona. Este dato nos ubica en una posición vanguardista respecto al manejo de las adicciones en prisión.

El presente artículo persigue cuatro objetivos. Primero, deseamos presentar y discutir el desarrollo y la pertinencia del PTAO; Segundo, queremos describir el contexto en el cual se desarrolla el mismo; Tercero, discutiremos las expectativas de diversas organizaciones referente a la inclusión de familiares en el tratamiento para superar la o las adicciones (estándares de cuido); Y, cuarto, el artículo persigue identificar y discutir las necesidades de familiares de confinados, según reportadas en la 
literatura, particularmente las necesidades de aquellos confinados que participan en programas de tratamiento para el abuso y dependencia de opiáceos en instituciones penales en la Isla. Al final del artículo planteamos algunas implicaciones de este fenómeno para la práctica directa e indirecta del trabajador social y presentamos algunas conclusiones generales y recomendaciones.

\section{Definición y Magnitud del Problema:}

En Puerto Rico existe consenso en que la violencia es uno de los principales problemas de salud pública y en que el tráfico de drogas ilegales es uno de los principales factores relacionados con diversas manifestaciones de esta violencia, entre ellos, los homicidios y la criminalidad (Santiago-Negrón, 2007; Santiago-Negrón \& Albizu, 2001). Sin embargo, cuando examinamos este fenómeno de cerca, encontramos que la cantidad de personas que cumplen con criterios de abuso y dependencia a drogas ilegales en la Isla es mínima, comparado a la población total que es de cerca de 4 millones de habitantes (Colón, Marrero, Robles, López \& Ramos, 2002). Este dato, sugiere que la violencia relacionada a las drogas parece guardar una relación más estrecha con el carácter ilegal de las mismas, su cultivo, distribución y venta, y con el contexto en el cual se utilizan, que con el consumo en sí. Lamentablemente, la escasez de tratamiento efectivo para las diversas adicciones y poblaciones afectadas por el abuso y dependencia a sustancias legales e ilegales en la Isla agravan este ya complicado panorama.

Un estudio reciente realizado en Puerto Rico (Colón, Robles, Cabassa \& López, 2002) estimó que al menos 178 mil adultos cumplen con criterios de dependencia (7.1\%) a alcohol u otras drogas, y que de estos, $93 \%$ no reciben tratamiento. Estimó, además, que cerca de 186 mil personas (7.5\%) cumplen con criterios de abuso a alcohol, y que $73 \%$ de estos no reciben tratamiento. Sobre el alcohol, el estudio demostró que el $90 \%$ de las personas que cumplen con criterios de dependencia y de abuso no reciben tratamiento para su condición (Colón y colegas, 2002). En general, sólo un $3.4 \%$ de los participantes había recibido algún tipo de tratamiento para la adicción a drogas en el sistema de salud física, salud mental, o en el sistema informal, al momento del estudio. 
A pesar de que existe evidencia de que la drogodependencia y el abuso de drogas ilegales en Puerto Rico ocurren equitativamente en la población general y que la proporción de personas afectadas por esta condición es comparable por escolaridad, ingreso familiar, estado laboral, estado marital y lugar de residencia (urbano vs. rural) (Albizu-García, Román Badenas, Caraballo, G., Hernández Vives y Caraballo J., 2005), los confinados con trastornos de drogas que proceden de los estratos socio-económicas más bajos están sobre-representados en la población penal del país, lo que sugiere que éstos están más propensos a ser encarcelados por su adicción que a recibir tratamiento para su condición. Albizu y sus colegas (2005) concluyen que existe relación entre el encarcelamiento y padecer de trastornos de drogas ya que las personas con abuso y/o dependencia a drogas están sobre-representadas en los penales del país en una proporción que es 10 veces mayor al de la población general. En el estudio de Albizu et al. (2005), la prevalencia de abuso o dependencia a drogas ilícitas entre varones encarcelados fue de $19.19 \%$ (abuso) y 31.39 (dependencia), mientras que para las mujeres encarceladas fue de $22.27 \%$ (abuso) y $40.45 \%$ (dependencia).

Un estudio de necesidades realizado por Colón, et al. (2001) sobre el Departamento de Corrección y Rehabilitación reveló que de una muestra de admisiones a esta agencia, 62\% tenían un trastorno de uso de substancias y un $50 \%$ tenían otro trastorno psiquiátrico. Las estadísticas de este Departamento y el estudio de Albizu et al (2007) estiman que un $80 \%$ de los confinados están sentenciados por delitos relacionados a trastornos de uso de substancias y que el $20 \%$ de la población estaba usando substancias activamente en la cárcel al momento del estudio, lo cual equivale a 2,400 personas. En el 2003 , un $49 \%$ de los confinados eran positivos al virus de la hepatitis $\mathrm{C}$ (VHC) en comparación a $6.9 \%$ de la población residente de San Juan (Pérez, Suárez, Torres, Román, \& Colón, 2005) y en el 2004, Salud Correccional reportó una prevalencia de $10 \%$ de positivos al virus de inmunodeficiencia adquirida (VIH).

No es sorprendente observar que, en el 2001, un estudio realizado por "CASA" (Center on Addiction and Substance Abuse, Columbia University), concluyó que el gobierno utiliza $\$ 277.00$ por cada contribuyente para castigar a la persona adicta, mientras que para prevenir, investigar y ofrecer tratamiento sólo utiliza diez $(\$ 10.00)$ dólares por cada contribuyente. En 1998, PR pagó cerca de 360 millones para administrar el encarcelamiento de adultos con drogodependencia $(86.3 \%$ de su 
presupuesto). De éstos, 111 millones se utilizaron para encarcelar a menores (74.4\%), y 29 millones se utilizaron en gastos de corte $(86 \%)$ (C.A.S.A., 2001).

La tasa de encarcelamiento en PR es de más de 350 personas por 100,000 habitantes, superada solo por EEUU, Rusia y un pequeño grupo de países (World Prison Population, 2003). Nuestro sistema correccional es sumamente complejo. El mismo cuenta con una población penal de cerca de 15 mil confinados y con 49 facilidades correccionales para confinados de custodias máxima, mediana y mínima. Durante el mes de junio de 2006, el sistema tuvo bajo su control un promedio de 14,276 personas, divididos entre 12,002 sentenciados y 2,274 sumariados (personas encarceladas no sentenciadas). De éstos, 439 eran jóvenes-adultos y 465 eran mujeres (Departamento de Corrección y Rehabilitación, 2006).

Si se mantiene el ritmo de crecimiento actual de la población carcelaria en Puerto Rico, en el 2020 se estima que habrá un total de 25,000 personas en prisión y se tendrán que habilitar 10,000 espacios carcelarios adicionales a un costo billonario. El costo actual aproximado de $\$ 40,000$ por confinado por año, se estima que en 2020 llegara a $\$ 58,400$ (Lcdo Pereira, entrevista El Nuevo Día, 20 de julio de 2006). Si consideramos, además, que la prevalencia de individuos co-infectados en la población confinada de PR es de un 32\% (Albizu et al., 2005) y que los costos anuales de estas condiciones son considerables (un paciente VIH+ gasta unos $\$ 14,000$ anuales, si ha desarrollado SIDA cuesta unos $\$ 34,000$ al año y el tratamiento de hepatitis cuesta unos $\$ 17,000$ ), en el 2020 se gastarán 1.5 billones de dólares en el DCR, más del doble del gasto actual.

Todo este panorama sugiere, entre otras cosas, que debemos invertir en la prevención de trastornos de uso de sustancias y debemos atender con efectividad y prontitud las necesidades de una población cautiva enferma que, de no ser atendida, re-ingresará a la sociedad con más enfermedades y mayor cronicidad que cuando entró al sistema carcelario, con grandes posibilidades de delinquir para llenar las necesidades de su adicción. En Estados Unidos, la literatura indica que aquellos confinados que no han recibido tratamiento para su dependencia a heroína suelen recaer antes del mes de la excarcelación (Kinlock, Gordon, Schwartz, O'Grady, Fitzgerald \& Wilson, 2007; Maddux \& Desmond 1981) y que esta recaída generalmente se acompaña de un aumento en la actividad delictiva 
(Chaiken \& Chaiken, 1990; Kinlock, O'Grady \& Hanlon, 2003), un aumento desproporcionado en el riesgo de infección con VIH (Chitwood, Comerford \& Weathersby, 1998; Inciardi, McBride, Duane \& Surrat, 1998), de hepatitis B y C (Edwin, 2002), muerte por sobredosis (Mark, Woody, Juday \& Kleber, 2001) y re-encarcelamiento (Hanlon, Nurco, Bateman \& O'Grady, 1998; Substance Abuse and Mental Health Services Administration, 2000a).

Tradicionalmente, las adicciones son vistas como deficiencias que surgen de fallos en la comunicación o funcionamiento de la familia. En este caso, al igual que con otros problemas sociales, la familia ha sido tratada como el 'sumidero' que explica las deficiencias de cada uno de sus miembros, incluyendo su comportamiento adictivo, su comportamiento delincuente y confinamiento. Bajo este enfoque, la familia es vista como un enemigo pues se entiende que desde allí proviene la raíz del probiema. Ante este enfoque patológico, la modalidad de terapia individual ha sido la preferida, con una tendencia marcada a excluir del tratamiento a la familia de la persona adicta.

Estudios recientes han ayudado a ampliar la forma de entender las adicciones. Existe evidencia que la adicción es una enfermedad crónica, progresiva y recurrente del cerebro, influenciada por factores genéticos, neurobiológicos, psicosociales y ambientales; que la misma se caracteriza por la pérdida de control sobre el uso de la substancia ya que el uso es compulsivo a pesar de consecuencias adversas; y que muchas veces se caracteriza por la insidia o ansias de uso (cravings) intolerables (Nestler \& Malenka 2004; Organización Mundial de la Salud 2004). En ésta perspectiva, las adicciones son vistas como condiciones primarias de salud lo cual causa problemas en la comunicación y el funcionamiento familiar. Ante este enfoque, la familia es vista como un aliado para el proceso de tratamiento y recuperación y no como un enemigo. La modalidad de terapia individual es utilizada como herramienta de apoyo, pero se promueve la integración de familiares a través de terapia de familia, terapia de grupo y/o terapia individual. La familia no es culpabilizada ni estigmatizada, sino que es atendida e integrada al proceso de tratamiento y recuperación de la persona con trastornos de uso de substancias (Van Wormer \& Rae Davis, 2003). 


\section{¿Por qué es pertinente desarrollar un PTAO en prisión?}

El Programa de Tratamiento de Agonista Opiáceos (PTAO) es un programa en desarrollo bajo el sistema carcelario de Puerto Rico el cual ofrecerá tratamiento farmacológico para la adicción a opiáceos con metadona, inicialmente, y eventualmente con buprenorfina. Se le conoce usualmente como 'Programa de Mantenimiento con Metadona' porque este nombre excluye la posibilidad de utilizar otros fármacos disponibles para esta condición.

La adicción a opióides es particularmente insidiosa, ya que el cerebro produce sus propias substancias opióides (endorfinas) que son vitales para su supervivencia. Los agentes opiáceos (heroína) 'engañan' al cerebro, ya que responden como si fueran biológicamente esenciales. Una vez la adicción se instaura, la química cerebral se desbalancea y la persona es física, emocional y mentalmente disfuncional a menos que continúe usando la substancia opiácea (heroína) regularmente. Estos trastornos son severos y duraderos y permanecen meses o años luego del último episodio de uso de la droga. Así que, aún cuando se logre abstinencia al opiáceo, las recaídas son comunes sin tratamiento (Nestler \& Malenka 2004, O.M.S. 2004).

Aunque existe una extensa experiencia mundial en la provisión de tratamiento farmacológico con metadona en escenarios correccionales (Jurgens 2004; McSweeney, Turnbull \& Haigh 2002), en EEUU, la experiencia no es tan abundante. En un estudio piloto de 1969, llevado a cabo en la ciudad de Nueva York, Dole tomó 12 confinados y los inició en metadona diez días antes de su excarcelación, refiriéndolos a un programa en comunidad. A los 7-10 meses de libertad, sólo 3 habían sido reencarcelados aunque ninguno había recaído en heroína. Del grupo control de 16 que no recibieron tratamiento, todos habían regresado a prisión. Desde el 1987 existe un programa en la cárcel de Riker's Island en Nueva York (Programa KEEP por sus siglas en inglés) que inicia a pacientes heroinómanos en metadona y continúa el tratamiento a pacientes que provienen de programas de metadona de la comunidad, refiriéndolos a seguimiento al ser excarcelados. Luego de 11 años, los hallazgos sugieren que el tratamiento de mantenimiento con metadona (MMT por sus siglas en inglés) en la cárcel facilita el tratamiento en la comunidad al ser excarcelados y también previene la re-encarcelación (Tomasino, Swanson, 
Nolan \& Shuman, 2001). El estudio más reciente de costo-efectividad, que toma en consideración el impacto de por vida ("lifetime impact") de la criminalidad y encarcelamientos potenciales, desempleo, utilización de servicios de salud y la posible necesidad de múltiples episodios de tratamiento, encontró una relación de costo-beneficio de cerca de $\$ 38.00$ por cada $\$ 1.00$ que se invierte en el tratamiento con metadona (Zarkin et al 2005).

En un estudio piloto de tratamiento de mantenimiento con metadona llamado Acuerdo de Superación en la prisión de Las Malvinas en Puerto Rico, se concluye que el consumo de heroína en los pacientes tratados se redujo en un $94-95 \%$ y que la participación en el programa fue asociado con una mayor aceptación del mantenimiento con metadona (Heimer, Catania, Newman, Zambrano, Brunet \& Marti Ortiz, 2006). El personal de la prisión y el Estado apoyaban el programa. Este proyecto fue impulsado y coordinado por la entonces existente Oficina de Control de Drogas como parte de su objetivo de expandir acceso a tratamiento. Sin embargo, el programa no ha sido expandido significativamente en los últimos años.

El Departamento de Corrección y Rehabilitación, además del programa de metadona mencionado ofrece la oportunidad de servicios de desintoxicación a los confinados con medicamentos no opiáceos y ofrece referidos a programas de comunidades terapéuticas. En este contexto, la 'detoxificación' de opiáceos (restaurar el cerebro a sus niveles de tolerancia a la droga del inicio de la adicción) es un tratamiento altamente inefectivo ya que trastornos psicológicos y la insidia a opiáceos (cravings) permanecen arraigados por un periodo indefinido de tiempo, lo cual hace que los deslices y recaídas sean mayormente inevitables (Maremmani, Matteo \& Lovrecic, 2004). La abstinencia a opiáceos y las terapias 'libres de drogas' pueden constituir tratamientos parciales y pueden posiblemente reducir daño cerebral adicional, pero no estabilizan el desbalance químico causado por la adicción a opiáceos que permitan un funcionamiento cerebral más normalizado. La experiencia del DCR sugiere resultados subóptimos en las modalidades de intervenciones sin base farmacoterapéutica

El sistema de corrección en Puerto Rico tiene un enorme problema ya que $66 \%$ de sus confinados han estado en el sistema anteriormente porque, al no ser rehabilitados, vuelven a ser apresados rápidamente. Sabemos que los trastornos adictivos y trastornos mentales son las dos condiciones de 
mayor prevalencia dentro del sistema correccional en la Isla y que la dependencia a heroína que no ha sido tratada resulta ser en gran parte responsable de esta reincidencia (Albizu et al, 2005). La revisión de literatura mostró que, tanto en el DCR como en otros sistemas correccionales en EEUU y Europa, el tratamiento con agonistas opiáceos iniciado en el sistema correccional y continuado en comunidad una vez los confinados son liberados reduce significativamente la reincidencia, reduce en un $50 \%$ el abuso de sustancias, reduce la actividad criminal hasta en un $80 \%$ y reduce en un $64 \%$ los arrestos relacionados a sustancias (National Institute on Drug Abuse, 2007).

Posiblemente la mayor ventaja del MMT en el entorno correccional es que se pueden convertir en escenarios terapéuticos para comenzar ofensores en tratamientos que pueden ayudar a promover estabilización duradera de su enfermedad cuando regresen a la comunidad. Los controles del ambiente carcelario pueden ayudar a superar la falta de adherencia que a veces causan fracasos en los tratamientos en la comunidad (Maremanni et al., 2004). Otro beneficio es que ayuda a mejorar la salud pública dentro y fuera del escenario carcelario. El escenario carcelario también provee una importante oportunidad para incorporar confinados con adicción a heroína a tratamiento, ya que muchos de ellos no han recibido tratamiento durante el encarcelamiento o en la comunidad (Brown \& Leedle, 1994; National Institute on Drug Abuse, 1999).

El tratamiento con agonistas opiáceos es uno de los más reglamentados del sistema en EEUU y por lo tanto en nuestro país, que sigue las pautas de ese país, lo regula el "Substance Abuse and Mental Health Services Administration's" (SAMHSA), el Departamento de Salud Estatal (ASSMCA) y el Departamento de Justicia Federal (DEA). Puesto que la adicción es una de las condiciones de salud que más prejuicio o estigma levanta en la ciudadanía, muchas entidades promueven esta visión prejuiciada, incluso dentro de las autoridades sanitarias y de la comunidad que forma parte del sistema de tratamiento de adicción del país. Dentro de la adicción, a su vez, el tratamiento con agonistas opiáceos tiene una camada adicional de prejuicio. Este prejuicio se refleja en aquel nivel de reglamentación exagerado, el cual puede explicarse por el desconocimiento de la relativa inocuidad de estos medicamentos y su alta efectividad. También se manifiesta en los recursos asignados para esta modalidad, los cuales son insuficientes para cubrir las necesidades de la población general 
y de los confinados y confinadas. En la Isla, sólo alrededor del 10\% de las personas que necesitan tratamiento con agonistas opiáceos lo obtienen (Colón et al., 2001).

En términos generales, existe mucha resistencia en Puerto Rico a los programas que ofrecen tratamiento farmacológico con metadona, Esta resistencia está basada, muchas veces, en mitos que se han propagado através de los años (CIES, 2007). Entre los más comunes está la aseveración: "La metadona es cambiar una droga por otra." Este tipo de expresión refleja total desconocimiento del efecto farmacológico de la metadona (igual se aplica a la buprenorfina) pues la metadona se toma por boca, no se inyecta; tiene un efecto que dura 24 horas y no 6 horas como la heroína; $\mathrm{y}$, en dosis de mantenimiento $\mathrm{y}$ por su acción de lenta instauración, la metadona no produce euforia como la heroína. Otro mito comúnmente citado es: "la metadona debilita los huesos y hace caer la dentadura." Esto es falso ya que la metadona no tiene que ver con los huesos. Si una persona en tratamiento con metadona tiene dolor en los huesos y en los músculos, tal vez sea debido al síndrome de retirada que experimentan los pacientes al irse retirando del consumo de heroína y que se van 'enfermando'. Tampoco la metadona afecta los dientes; el paciente que tiene dependencia a heroína cuida muy poco su higiene oral además de que por ser la heroína un analgésico, los síntomas de dolor de patología dental van a ser opacados por la heroína.

Otro mito generalizado sobre la metadona es que no funciona. La metadona, en conjunto con intervenciones psicosociales adecuadas, es el tratamiento más efectivo conocido para la dependencia a heroína. Sin embargo, es muy importante que el tratamiento sea implementado de forma adecuada, o sea, en una dosificación que atienda las necesidades del paciente (que elimine el síndrome de retirada y que suprima los deseos de uso o insidia) y por el tiempo necesario en el que el paciente se este beneficiando del tratamiento, sin ningún limite arbitrario de duración. $\mathrm{Si}$ los medicamentos antidiabéticos, antihipertensivos, antisicóticos y de otras condiciones crónicas no se limitan, ¿por qué se hace con la metadona, sino es por prejuicio?

Existe abundante evidencia desde los años 60 de que el tratamiento con agonistas opiáceos (metadona, LAAM y, más recientemente, buprenorfina) son altamente eficaces en reducir la adicción a la heroína, en reducir el riesgo de infección de VIH y otras enfermedades infecciosas, y en reducir 
la actividad delictiva y el re-encarcelamiento, evidencia que fue endosada por el Instituto de Medicina en 1995 y por el panel de expertos de los Institutos Nacionales de Salud (National Institute of Health, 1997) en su "Consensus Statement" (1997). Este último, específicamente, recomendó el acceso a tratamiento con metadona para todas las personas dependientes de opiáceos bajo jurisdicción legal.

La creación del Programa de Tratamiento de Opiáceos (PTAO) en una prisión en Puerto Rico, el cual cuenta con el apoyo incondicional de su Secretario, Lic. Miguel A Pereira, ha requerido reuniones educativas con los diversos grupos de interés para clarificar dudas e informar lo que cada grupo debe hacer para que el mismo logre concretarse. El programa tiene como metas reducir la reincidencia dentro del sistema correccional, proveyendo tratamiento farmacológico comprensivo a 200 pacientes varones con dependencia a heroína dentro del sistema correccional de PR. Además, persigue reducir el contagio con enfermedades infecciosas debido al uso de heroína con parafernalia inyectable contaminada.

\section{Expectativas sobre la inclusión de la familia del confinado en PTAO}

Debido al ambiente restrictivo del sistema carcelario, es importante reflexionar sobre por qué, cómo, cuándo y dónde podrían integrarse los familiares de confinados al tratamiento con metadona en prisión. Existe literatura sobre cómo y cuánto se afecta la familia de una persona que padece de drogodependencia, ya sea a drogas legales o ilegales, y sobre el rol de la familia en el proceso de tratamiento y recuperación de las adicciones (Var Wormer \& Rae Davis, 2003). Sin embargo, la literatura local sobre estos temas es escasa.

Sabemos que usualmente las familias sufren cuando alguno de sus miembros desarrolla abuso o dependencia a sustancias y que este sufrimiento suele agravarse cuando la familia también tiene que lidiar con el encarcelamiento de la persona adicta. Sin embargo, las respuestas particulares de cada familia ante el progreso e intensificación del comportamiento adictivo podrían ser variadas. Entre las respuestas más comunes está el culpabilizar a la persona adicta por su adicción, ocultar objetos, encubrir el comportamiento adictivo, unirse al comportamiento adictivo, alejarse del adicto/a, y/o evitar la presencia de personas extrañas a 
la familia (Var Wormer \& Rae Davis, 2003). El encarcelamiento agrava una situación ya de por sí estigmatizante y el regreso del confinado al hogar, sin haber recibido tratamiento para su adicción, representa un reto aún mayor para la familia y la sociedad. Aún cuando la persona se recupera, tanto el individuo como la familia tienen que activar nuevamente sus procesos adaptativos para esta nueva situación.

Durante tres años (1996-99), el "Center for Substance Abuse Treatment" (CSAT, 2007) desarrolló unas Guías de Acreditación para Programas de Tratamiento de Agonista Opiáceos, las cuales fueron revisadas y publicadas nuevamente en julio de 2007. Estas guías se fundamentan en protocolos conocidos como "Treatment Improvement Protocol (TIP)", en trabajos producidos por paneles de expertos, en investigaciones de campo y otras fuentes pertinentes. Las guías de CSAT responden a los estándares de acreditación de programas de tratamiento de agonista opiáceos establecidos por el SAMHSA; mientras que los estándares de SAMSHA responden a los estándares federales para estos programas que se encuentran en el Título 42, Parte 8 del "Code of Federal Regulations (CSAT, 2007)."

Al examinar estos estándares para determinar si contemplan o no la integración de la familia en el tratamiento con metadona encontramos que, en efecto, existen criterios que promueven y protegen la participación de familiares en esta experiencia. SAMSHA, incluso, recomienda que se amplíe la definición de 'familia' para que incluya otras personas significativas para el paciente en el tratamiento, ya que muchas veces, por razones mencionadas anteriormente, es sumamente difícil, si no imposible, integrar a los familiares al tratamiento del adicto. $\mathrm{Al}$ respecto, las guías de acreditación indican (CSAT, 2007). De igual forma, el "Estándar H", por ejemplo, identifica áreas específicas sobre el historial familiar que no deben de ser pasadas por alto durante la evaluación psicosocial del paciente de metadona, entre ellas, varias características sociodemográficas de los hijos y variables relacionadas al historial médico- familiar del paciente: Por su parte, el "Estándar \# D.d", discute los derechos del paciente y establece en qué condiciones los familiares podrán representar al paciente $\mathrm{y}$ tomar decisiones sobre su tratamiento.

Los estándares de acreditación de SAMSHA también proponen atender las necesidades del paciente, en este caso del confinado, respecto a sus roles y funciones en la familia. El Estándar Q, por ejemplo, discute consideraciones importantes en la planificación del tratamiento y presenta 
un listado de necesidades que, de atenderse en el paciente, le ayudarían a fortalecer y solidificar lazos con su familia. Entre estas, mencionan la necesidad de proveer talleres educativos sobre destrezas de crianza, ofrecer servicios de salud reproductiva y atender otras necesidades de salud mental del paciente. Este estándar, también recomienda que se atiendan las necesidades de servicios de los hijos de los pacientes de metadona, especialmente necesidades relacionadas a su salud mental y necesidades cognitivas.

El National Commission on Correctional Health Care (NCCHC, 2004) también tiene sus estándares para el tratamiento de la adicción a opióides en el sistema carcelario. Aunque ningún estándar identifica directamente a la familia del confinado como un componente esencial en el tratamiento de la adicción, tampoco obstaculizan la inclusión de los mismos. De hecho, la evaluación psicosocial comprensiva que requiere NCCHC en sus estándares permite y promueve la evaluación de sistemas sociales relacionados al confinado, entre ellos la familia. Aquellos programas en el sistema carcelario que integran la familia en el tratamiento del confinado son considerados como programas que exceden el estándar de cuido, lo cual es muy positivo.

El National Quality Forum (2007) desarrolló y publicó unos estándares que voluntariamente y por consenso cientos de organizaciones acogieron recientemente en los Estados Unidos respecto al tratamiento del abuso y dependencia a opiáceos. Cónsono con los estándares de acreditación de SAMSHA, este informe recomienda que cada paciente reciba una evaluación comprensiva de su condición, lo cual incluye el explorar las fuentes de apoyo familiar de cada paciente (recursos), las necesidades de este sistema respecto al paciente y el establecer planes de tratamiento que consideren el sistema familiar. Por su parte, y basados en la literatura sobre el tratamiento efectivo para la adicción a drogas y sobre comportamiento delictivo, el National Institute on Drug Abuse (NIDA, 2007) publicó trece estándares de cuido esenciales para la población confinada con trastornos de uso de sustancias. Aunque ninguno de estos estándares menciona directamente el componente familiar, varios estándares proveen espacio para la integración de este sistema al proceso de tratamiento y recuperación del confinado. Puesto que el tratamiento para la drogodependencia puede ser incorporado al sistema de justicia de varias formas (cortes de drogas, tratamiento durante probatoria, etc.), NIDA plantea en este documento la 
necesidad de que el sistema carcelario y el de justicia trabaje en colaboración con otras agencias gubernamentales que puedan complementar los servicios para el tratamiento de las adicciones, como servicios sociales y otros servicios de salud, etc.

El Programa de Tratamiento de Agonista Opiáceos (PTAO), tiene un componente psicosocial el cual incluye servicios de trabajo social. Estos servicios incluyen el realizar intervenciones para incluir a la familia del confinado en el tratamiento, en la medida de lo posible. Según establece la literatura, se espera que la integración de la familia al proceso de tratamiento y recuperación del confinado contribuya al éxito del tratamiento y aumente la retención del confinado en el mismo ya que se ha demostrado que el tiempo de retención en tratamiento se relaciona directamente con resultados positivos post tratamiento (Anglin \& Hser 1990; Nurco, Kinlock \& Hanlon 1994). El programa "Acuerdo de Superación" integró, en sus inicios, a los familiares de confinados el tratamiento y, aunque la experiencia fue exitosa, razones administrativas determinaron la terminación de estos servicios.

\section{Necesidades de familiares de confinados}

Para ésta sección se llevó a cabo una revisión de literatura basada en una búsqueda sistemática en la base de datos del Sistema de Bibliotecas de la Universidad de Puerto Rico, Recinto de Río Piedras. También se revisaron tesis disponibles en la Escuela de Trabajo Social y la Escuela Graduada de Administración Pública de la Universidad de Puerto Rico, y una tesis realizada en la Universidad Carlos Albizu. Además, se utilizaron algunas fuentes identificadas en el Internet.

Esta búsqueda sistemática resultó en la identificación de seis estudios relevantes para Puerto Rico relacionados al tema de investigación (Fuentes, et al., 1990; Fernández, 2002; De Jesús, 2002; Feliciano, 1996; Báez, et al., 1973). En sus estudios, De Jesús (2002) y Fernández (2002) presentan las relaciones de los confinados con su descendencia y familiares y discuten la falta de inversión de parte del gobierno para diseñar políticas públicas que ayuden a este sector. Un estudio de hace más de tres décadas buscaba determinar el funcionamiento social de las familias de confinados (Báez et al., 1973), mientras que un estudio más reciente examinó el impacto del confinamiento en las familias de los confinados (Fuentes, et al., 1990). 
Entre las debilidades encontradas en la literatura, se encontró que la mayoría de los estudios disponibles provenían de Estados Unidos. Los estudios hechos en Puerto Rico no son recientes y los resultados obtenidos de los mismos muestran una realidad muy distinta a la que presentan los estudios de Estados Unidos, Colombia (Moreno \& Zambrano, 2007) y Finlandia (Karsikas, 2005). Estos últimos muestran un descontento muy evidente por parte de lo familiares de los confinados con las visitas maritales y familiares en la institución; con la participación que se les da en el proceso de rehabilitación del confinado; y con la ayuda que reciben para contrarrestar los efectos del encarcelamiento en las familias, entre otros. En Puerto Rico, las investigaciones tienden a mostrar mayor grado de conformidad por parte de los familiares y el confinado con los servicios que reciben durante y después del proceso de encarcelamiento.

La mayoría de los confinados forman parte de un sistema familiar en el que el funcionamiento emocional, económico y social se ve interrumpido por la ausencia de ese miembro. Como la familia es un sistema donde todos tienen que trabajar conjuntamente para mantener el equilibrio, el confinamiento de uno de sus miembros afecta tanto al confinado como a su familia (Fuentes, et al., 1990). Una de las áreas que se impacta es el aspecto económico ya que muchas veces el individuo encarcelado era quien sostenía el hogar o contribuía significativamente con sus ingresos (Moreno \& Zambrano, 2007). Esta pérdida de ingresos implica mayores gastos gubernamentales pues aumenta la dependencia en estas instituciones. El estudio de (Arditti, Lambert-Shute, \& Joest, 2003) reportó que los padres confinados sufren las crisis económicas de sus familiares y el estigma social que estos experimentan.

Una entrevista realizada por la doctora Lowenstein a 118 esposas de confinados "demostró que no sólo la aportación económica del esposo encarcelado afecta la economía de la familia sino también la inclusión de nuevos gastos legales" (Travis, 2005:15). En otro estudio realizado en Estados Unidos, varias mujeres expresaron que las responsabilidades adquiridas desde el encarcelamiento de su familiar no les permitían tener tiempo ni para ellas mismas. La falta de tiempo se asociaba con el cuido de los niños y el empleo (la doble jornada) (Arditti; Lambert-Shute \& Joest, 2003). 
Los confinados y sus familiares sufren de estigma social como efecto del confinamiento (Santiago-Negrón \& Albizu-García, 2007; Varas-Díaz, Serrano-García \& Toro, 2004). La discriminación ante la sociedad y el trabajo muchas veces llevan al familiar al extremo de tener que negar la existencia del familiar encarcelado y cambiar de círculo social (Ligth, Campbell, 2006). Según esta fuente, los hijos e hijas de confinados mayormente sufren discriminación por parte de sus maestros, compañeros u otros miembros de la familia lo que les baja la autoestima y los hace vivir con vergüenza.

Para cualquier persona la separación de un ser querido es un proceso doloroso. Un divorcio, la muerte de algún familiar o amigo, la salida del hogar de alguno de los miembros o la encarcelación son asuntos que generan trauma y serios problemas emocionales. De acuerdo a la literatura revisada, el confinamiento de un familiar puede causar daños emocionales, los cuales pueden provocar comportamientos antisociales y otros problemas mentales (Wright \& Seymour, 2000). Según Wright y Seymour, el encarcelamiento evita que los hijos e hijas de confinados se desarrollen emocionalmente saludables, creando así una niñez con estrés y coraje contra su padre, madre o la sociedad misma. Las parejas de los encarcelados también se ven impactados no solo en el ámbito sexual sino también emocional por los sentimientos de impotencia ante el sistema de justicia, la soledad y confusión por la inocencia o culpabilidad del interno.

La percepción de los familiares sobre cómo pueden ellos involucrarse en el tratamiento del confinado, durante el confinamiento y al salir de prisión fueron áreas exploradas en la literatura. Muchas veces la falta de información los lleva a pensar que no pueden contribuir al proceso de rehabilitación del confinado. Autores como Karsikas (2005) y Arditti, Lambert-Shute \& Joest (2003), han coincidido en que es de suma importancia reemplazar las relaciones de los confinados entre sí por una relación entre familiares con confinados. De acuerdo a Moreno \& Zambrano (2007), científicos sociales han afirmado que los programas dirigidos hacia el confinado y sus familiares producen resultados positivos a nivel institucional, familiar y comunitario.

Cuando la persona confinada es excarcelada, los familiares muchas veces tienen la intención de apoyarle pero sufren de escasez de recursos para aportar en el proceso (falta de vivienda apropiada, empleo para el confinado, comida, etc.). Se ha demostrado que los presos tienen muchas 
expectativas en cuanto a la ayuda que les darán los familiares una vez salgan de prisión. Según señala Visher et al. (2004), un gran porcentaje espera que algún familiar los ayude económicamente, les provean un techo o les ayude a conseguir un empleo. Sorprendentemente los confinados entrevistados para este estudio expresaron que no solo el apoyo económico por parte de sus familiares era de suma importancia, si no también el apoyo emocional para no volver a caer en prisión. Este estudio concluye que el tipo y el nivel de ayuda que les brinden los familiares de los ex confinados, ya sea emocional, económica, de vivienda o transportación, influye en cómo el confinado triunfa o fracasa luego de su excarcelación.

El efecto negativo que puede traer el confinamiento en la relaciones del confinado con sus hijos es otra área de gran preocupación en la literatura. Este tema es preocupante tanto para las familias de los confinados como para la persona confinada. Algunos estudiosos como Caña \& Caña (1998) citado en Travis (2005), han estimado que cerca de 10 millones de niños en Estado Unidosse ven afectados por la reclusión paternal en los sistemas carcelarios. El estudio realizado por Caña \& Caña encontró que cerca de 5 millones de niños menores de 18 años en ese país tiene uno o ambos padres bajo alguna supervisión del sistema penal, 1.5 millones tiene por lo menos uno de sus padres en la cárcel y unos 5 millones de niños han tenido en el pasado a alguno de sus padres bajo supervisión del sistema penal (Travis, 2005). Cuando las familias le ocultan la realidad del confinamiento de su padre o madre para evitarles sufrimiento, esto a su vez provoca un ciclo de mentiras que puede crear una confusión emocional en el menor y tener graves efectos secundarios. Sin embargo, cuando los menores conocen sobre el confinamiento y visitan las instituciones, se les debe prestar mucha atención y apoyarlos en el ajuste a esta situación. Muchas instituciones carcelarias tienen normas que hacen más difícil el contacto de los hijos e hijas con sus progenitores, como la edad que deben de tener para asistir a la cárcel, quien los tiene que acompañar, las cantidades de llamadas telefónicas que pueden hacer, los altos costos de estas llamadas (Travis, 2005). Un estudio realizado en Puerto Rico (Feliciano, 1996) reflejó que los hijos e hijas sufren por el rechazo social y las comparaciones con su progenitor. Esto a su vez tiene como consecuencia bajo rendimiento académico, ansiedad, depresión, inmadurez, conducta agresiva y otros problemas emocionales. Los familiares a cargo de esos niños y niñas tienen la responsabilidad de reeducarlos para afrontar con madurez la situación del padre o madre que esta encarcelado y la institución, a su vez, tiene la 
responsabilidad de facilitar el desarrollo de vínculos familiares satisfactorios.

Según Harrison (2002), cuando los abuelos y abuelas quedan al cuidado de los hijos e hijas de los confinados, el problema puede complicarse pues muchas veces estos son de edad muy avanzada, lo que limita la manera en que pueden asumir esta nueva responsabilidad y sus implicaciones económicas. Muchas veces no están preparados para tomar nuevamente responsabilidades relacionadas a la crianza de menores y sufren de muchos problemas de salud. Las normas que rigen el sistema penitenciario y el trato que a veces reciben los familiares del personal de la institución son aspectos que también pueden afectar la relación de los familiares con el confinado o confinada. Las distancias geográficas por confinamientos y traslados lejos del área geográfica donde residen los familiares dificultan las visitas de estos, encontrándose casos donde la persoiıa confinada no recibe visitas durante su encarcelamiento. Por otra parte, las visitas a las instituciones penales no son muy atractivas porque, entre otras cosas, el familiar que visita se expone a ser registrado indebidamente por el personal de la institución, podría pasar muchas horas esperando a la persona confinada y se expone a recibir malos tratos e insultos por parte de otros visitantes o personal de seguridad, entre otros (Moreno \& Zambrano, 2006) Todas estas situaciones son disuasivos que fomentan la desintegración del vínculo familiar.

Cuando las investigadoras examinaron si existían publicaciones sobre las necesidades de familiares de confinados que reciben tratamiento en prisión para su trastorno de abuso o dependencia a drogas, no encontraron datos relevantes. Dos estudios de la Dra. Carmen Albizu y sus colegas (2005, 2007) examinaron algunas características relacionadas a la composición familiar de confinados que recibían tratamiento con metadona o buprenorifina, pero no se identificaron las necesidades de este grupo en los artículos ya que este no era el tema de los mismos. Tampoco encontramos estudios que examinaran las necesidades educativas de los familiares respecto al tratamiento con opiáceos y respecto al proceso de re-inserción del confinado en la comunidad.

\section{Resumen y conclusiones}


El Programa de Tratamiento de Agonista Opiáceos (PTAO) del Departamento de Corrección y Rehabilitación en Puerto Rico está localizado en la Cárcel Regional de Bayamón, Módulo 1072. Actualmente reciben servicios cerca de 20 confinados, cantidad que debe aumentar durante los próximos meses hasta llegar al cupo de 150 pacientes. El PTAO de PR es el único programa en prisiones en el territorio de Estados Unidos ya que en ese país sólo los confinados que esperan su sentencia (sumariados) o en programas de desvío reciben tratamiento con metadona en algunos estados. Este dato nos ubica en una posición vanguardista con respecto al manejo de las adicciones en prisión.

Un reto importante del programa será identificar claramente las necesidades de los familiares del confinado y qué mecanismos parecen funcionar mejor para integrarles al tratamiento. La ampliación del concepto 'familia' que sugieren los estándares de acreditación de SAMSHA es un ámbito sobre el cual también estaremos reflexionando con detenimiento ya que el ambiente restrictivo de la prisión podría obstaculizar la inclusión de personas que no son familiares del confinado, aunque éste los considere como tal. Un estudio de necesidades utilizando el método de grupos focales será conducido por las autoras para explorar estas dos áreas y para identificar las necesidades educativas de los familiares respecto al tratamiento con opiáceos y respecto al proceso de reinserción del confinado en la familia y en la comunidad.

La profesión de trabajo social responde a valores y principios éticos claros. Estos principios y valores deben ser vistos como una de las justificaciones más importantes para que el trabajador social se involucre en los procesos de decriminalización de las adicciones. En el aspecto clínico, por ejemplo, los estándares de acreditacion de National Comisión on Correccional Health Care (NCCHC, 2004) señalan que el desarrollo del plan de tratamiento de un confinado es decisión del médico y del personal de salud. Sin embargo, nuestro programa en PR ha adoptado el modelo canadiense en el cual el confinado participa activamente en la confección de su plan de tratamiento, lo cual es cónsono con los estándares de cuido sugeridos por SAMSHA y con los valores del trabajador social. Esto, unido al énfasis para trabajar con las fortalezas del confinado y su familia en vez de sus defectos, son ejemplos concretos sobre cómo el trabajador social puede 
utilizar su base filosófica y valorativa para continuar decriminalizando una enfermedad que ha sido históricamente criminalizada.

En el nivel mezzo, la inclusión de los familiares de confinados en el proceso de recuperación de este promueven que el trabajador social refine y utilice sus conocimientos y destrezas para llevar a cabo intervenciones con la familia y para abogar por estos en diversos foros. Una vez el confinado esté en la comunidad, profesionales de trabajo social de diversos sectores pueden contribuir a disminuir y a lidiar efectivamente con el estigma que sentirá éste y su familia por el encarcelamiento, por los trastornos adictivos y por el tratamiento con metadona recibido en prisión. Para esto, sin embargo, la profesión tiene que promover la educación en este campo del saber. El nivel macro es sin duda uno de los niveles donde la profesión de trabajo social puede aportar más a la decriminalización de las adicciones. En este nivel, el desarrollo de políticas de drogas vanguardistas y la eliminación de políticas de drogas punitivas existentes son dos mecanismos fundamentales para promover la adopción de un modelo más cónsono con los valores y principios de esta profesión, como lo es el modelo de salud pública.

La educación es una herramienta básica para lograr cambios en el ser humano. Aunque el adiestramiento no garantiza la adopción de una conducta en particular, ayuda a facilitar la transformación del individuo. Por lo tanto, es imperante examinar las necesidades educativas del personal del PTAO en prisión sobre el tema de las adicciones, incluyendo el personal de custodia, y las necesidades educativas de los confinados y sus familiares. Todos estos esfuerzos, unidos a la alta efectividad del tratamiento farmacológico con metadona en combinación con servicios psicosociales, producirán grandes cambios en el entorno social. Específicamente, en los próximos cinco años debemos comenzar a ver una reducción significativa en la tasa de contagio de $\mathrm{VIH}$, hepatitis $\mathrm{C}$ y otra enfermedades entre los confinados y en la comunidad y una disminución, también significativa, en la tasa de reincidencia carcelaria. ¡Así sea!

\section{Referencias}

Albizu-García, Carmen; Román, Luis; Caraballo Correa, Glorimar; Rivera, Santiago; Hernández Viver, Adriana \& Caraballo, José. (2005). Estudio de Necesidades de Tratamiento para Abuso/Dependencia a drogas y Prevención de Hepatitis B/C en las prisiones de Puerto Rico. 
San Juan: Centro de Investigación y Evaluación Sociomédica, Universidad de Puerto Rico.

Albizu García, Carmen; Caraballo Correa, Glorimar; Hernández Viver, Adriana; Kimlock, Timothy W.; Gordon, Michael S.; Antron Avila, Cristóbal; Colón Reyes, Ivette \& Schwartz, Robert. (2007). Buprenorphine-Naloxone Treatment for Pre-release OpioidDependent Inmates in Puerto Rico. Journal of Addiction Medicine, 1(3), 1-6.

Arditti, Joyce A.; Lambert-Shute, Jennifer \& Joest Arditti., Karen. (2003). Saturday Morning at the Jail: Implications of Incarceration for Families and Children. Family Relations, 52(3), 195.

Baez, Iris; Bernabe, Rosa M.; Colón María J.; Cruz, Sylvia \& Cuadrado, María A. (1973). Estudio sobre familias de confinados. Tesis de maestría no publicada, Universidad de Puerto Rico en Río Piedras.

Brown, Barry S. \& Needle, Richard H. (1994). Modifying the process of treatment to meet the threat of AIDS. International Journal of Addictions, 13(29), 1739-1752.

Center for Substance Abuse Treatment. (2005). Medication-Assisted Treatment for Opioid Addiction in Opioid Treatment Programs. Treatment Improvement Protocol (TIP) Series 43. DHHS Publication No. (SMA) 05-4048. Rockville, MD: Substance Abuse and Mental Health Services Administration.

Center for Substance Abuse Treatment. (2007). Guidelines for the Accreditation of Opiod Treatment Programs. Center for Substance Abuse Treatment. Recuperado de: http://www.dpt.samhsa. gov/pdf/draft_accred_guidelines.pdf.

Center on Addiction and Substance Abuse at Columbia University (C.A.S.A.). (2001). Shoveling the Impact of Substance Abuse on State Budgets. New York: Columbia University.

Centro de Investigación y Evaluación Sociomédica (CIES). (2007). Mitos sobre el tratamiento con metadona. San Juan: Centro de 
Investigación y Evaluación Sociomédica. Recinto de Ciencias Médicas, Universidad de Puerto Rico.

Chaiken, Jan M. \& Chaiken, Marcia R. (1991). Drugs and crime. En Michael, Tonry, \& James Q. Wilson (Eds), Crime and Justice: A review of research. Vol 13, pp 584). Chicago: University of Chicago Press.

Chitwood, Dale D.; Comerford, Mary \& Weatherby, N. (1998). The initiation and use of heroin in the age of crack. Heroin in the age of crack-cocaine. Thousand Oaks, CA: Sage Publications.

Colón, Héctor M.; Robles, Rafaela; Cabassa, M. \& López, C.M. (2001). Needs Assessment Program: Need of Substance Abuse Services Among Correctional Inmates in Puerto Rico. Puerto Rico: Administración de Servicios de Salud Mental y Contra la Adicción (ASSMCA).

Colón, Héctor; Marrero, Carmen; Robles, Rafaela; López, C.M. \& Ramos, G. (2002). Puerto Rico's Substance Abuse Need Assessment Program - Household survey final results. Puerto Rico: Administración de Servicios de Salud Mental y Contra la Adicción.

De Jesús, Giomar M. (2000). Diseño de una politica pública para los hijos $e$ hijas de padres confinados. Tesis de maestría no publicada. Universidad de Puerto Rico, Río Piedras.

Desmond, David P. \& Maddux, James F. (1981). Religious programs and careers of chronic heroin users. American Journal of Drug Alcohol Abuse. 8(1),71-83.

Departamento de Corrección y Rehabilitación de Puerto Rico. (2006). Perfiles de la Población. Portal Electrónico del DCR de PR. Recuperado el 4 de diciembre de 2007 de http://www.ac.gobierno.pr/Portal/DCR_docs.aspx.

Edlin, Brian R.(2002). Prevention and treatment of hepatitis $\mathrm{C}$ in injection drug users. Hepatology. 36(5-S1), 210-219. 
Feliciano, Isabel. (1996). Programa de adiestramiento para el fortalecimiento familiar con confinados adultos en Instituciones Penales. Tesis doctoral no publicada, Universidad Carlos Albizu, San Juan.

Fernández, Glorymar. (2002). Padres encarcelados. La percepción que tiene el confinado de su rol de padre, su interés de mantener relaciones filiales y la satisfacción con los servicios institucionales disponibles para la interacción familiar en la institución carcelaria de Bayamón a Mayo de 2002. Tesis de maestría no publicada. Universidad de Puerto Rico, Río Piedras.

Hanlon, Thomas E.; Nurco, David N.; Bateman, Richard.W. \& O'Grady, Kevin E. (1998). The response of drug abuser parolees to a combination of treatment and intensive supervision. The Prison Journal, 78(1), 31-44.

Heimer, Robert; Catania, Holly; Newman, Robert G.; Zambrano, John; Brunet, Arlyn \& Marti Ortiz, Arturo. (2006). Methadone maintenance in prison: evaluation of a pilot program in Puerto Rico. Drug and Alcohol Dependance. 83(2), 122-129.

Inciardi, James A.; McBride, Duane C. \& Surrat, Hilary L. (1998). The heroin street addict: Profiling a national population. Heroin in the Age of Crack-Cocaine. Thousand Oaks CA: SAGE.

Jurgens, Ralf. (2004). Is the world finally waking up to HIV/AIDS in prisons? A report from the 15th International Aids Conference. Infectious Disease in Correction Report. 7(9).

Karsikas, Vuokko. (2005). Estudio basado en el fortalecimiento del Trabajador Social. Tesis de la Facultad de Ciencias Sociales, Universidad de Helsinki.

Kinlock, Timothy W.; Battes, Ralf J.\& Schwartz, Robert. (2005) A novel opioid maintenance program for prisoners: report of post-release outcomes. American Journal of Drug and Alcohol Abuse, 31(3), 43354. 
Kinlock, Timothy W.; Gordon, Michael; Schwartz, Robert; O'Grady, Kevin; Fitzgerald, Terrence T. \& Wilson, Monique. (2007). A randomized clinical trial of methadone maintenance for prisoners: results at 1-month post-release. Drug Alcohol Dependance, 91(23), 220-227.

Kinlock, Timonty W., O'Grady, Kevin, \& Hanlon, T.E. (2003). Prediction of the criminal activity of incarcerated drug-abusing offenders. Journal of Drug Issues, 33(4), 897-920.

Maddux James F. \& Desmond, David P. (1981). Religious programs and careers of chronic heroin users. American Journal of Drug and Alcohol Abuse, 8(1),71-83.

Maremmani, Icro; Matteo, Pacini \& Lovrecic, Mercedes. (2004). Clinical foundations for the use of methadone in jail. Heroin Addiction \& Related Clinical Problems, 6(2-3), 53-70.

Mark, Tamy L.; Woody, George E.; Juday, Tim \& Kleber, Herbert D. (2001). The economic costs of heroin addiction in the United States. Drug and Alcohol Dependence, 61(2), 195-206.

McSweeney, Tim; Turnbull, Paul J. \& Hough, Mike. (2002). Review of criminal justice interventions for drug use in other countries. London: Criminal Policy Research Unit.

Morales, Alma R.; Pimentel, Vicky; Rodríguez, Leticia; Sánchez, Wanda; Sánchez, Delia E. \& Villanueva, Ivette M. (1990). El impacto del confinamiento en la familia del confinado. Tesis de maestría no publicada, Universidad de Puerto Rico en Río Piedras.

Moreno, Carlos \& Zambrano, Luis. (2006). Familias de internos $e$ internas: Una revisión de la literatura. Recuperado el 14 de julio de $2007 \mathrm{de} \mathrm{http://www.psicologiajuridica.org.}$

Mumola, Christopher. 2000. Incarceted parents and their children. Bureau of justice Statistics Special Report (Ncj 182335). Washington, D.C: U.S. Department of Justice. 
Naser, Rebeca \& Visher, Christy. (2006). Family members' experiences with incarceration The effect of incarceration and reentry. Western Criminology Review, 7(2), 20-31.

National Commission on Correctional Health Care. (2004). Standards for Opioid Treatment Programs in Correctional Facilities. Chicago, Ill: National Commission on Correctional Health Care.

National Institutes on Drug Abuse (NIDA). (1999). Principles of Drug Addiction Treatment: A Research-Based Guide. NIH pub \#99-4180. Recuperado el 15 de septiembre de 2007 de http://www.nida.nih.gov/PDF/PODAT/PODAT.pdf.

National Institute on Drug Abuse. (2007). Info Facts: Treatment for Drug Abusers in the Criminal Justice System. Recuperado el 20 de octubre de 2007 de

http://www.drugabuse.gov/ DrugPages/cj.html.

National Institute on Drug Abuse. (2006). Info Facts: Treatment Approaches for Drug Abuse. Recuperado el 9 de septiembre de 2007 de http://www.nida.nih.gov/Infofacts/TreatMeth.html

National Quality Forum (2007). National Voluntary Consensus Standards for the Treatment of Substance Use Conditions: Evidence-Based Treatment Practices A Consensus Report. Washington, D.C.: National Quality Forum, Inc. Recuperado el 14 de octubre de 2007 de http://www.qualityforum.org.

Negrón-Velázquez, Gisela. (2007a). Análisis del paradigma prohibicionista y el paradigma de salud pública aplicados al manejo de la drogadicción y criminalidad en Puerto Rico: Implicaciones para la práctica del trabajador social. En Dagmar Guardiola-Ortiz; Carmen Guemarez-Cruz \& Agnes Rivera-Casiano (Eds.), La Crisis Sostenida: Retos para la politica social y el trabajo social. (pp. 486-500). San Juan, Puerto Rico: Edil.

Negrón-Velázquez, Gisela. (2007b). Convergencias entre la perspectiva de fortalezas y la espiritualidad: Una perspectiva 
alterna para el tratamiento de las adicciones en Puerto Rico. Revista Análisis, VIII (1), 23-44.

Nestler Eric J. \& Malenka Robert C. (2004). The addicted brain. Science American. 290 (3), 78-85.

National Institute of Health. (1997-1998). Consensus Statement. Washington, D.C.: NIH. Recuperado el 18 de septiembre de 2007 de http://consensus.nih.gov/1997/1998TreatOpiateAddiction108html.htm

Organización Mundial de la Salud (2004). Informe sobre la salud en el mundo (1995- 2006). Recuperado el 21 de octubre de 2007 de http://www.who.int/whr/es/index.html

Pérez Pérez, Cynthia; Suárez, Erick; Torres, Esther A.; Román, Karisse \& Colón, Vivian. (2005). Seroprevalence of hepatitis C virus and associated risk behaviors: a population-based study in San Juan, Puerto Rico. International Journal of Epidemiology, 34(3), 593-599.

Santiago Negrón, Salvador \& Albizu García, Carmen. (2001). ¿Guerra contra las drogas o guerra contra la salud? Los Retos para la Salud Pública de la Política de Drogas de Puerto Rico. Puerto Rico Health Sciences Journal, 22(1), 49-61.

Santiago-Negrón, Salvador \& Albizu-García, Carmen. (2007). El impacto del estigma en el tratamiento de la dependencia a drogas ilegales. En Nelson Varas-Díaz \& Francheska Cintrón-Bou (Eds.), Estigma y Salud en Puerto Rico: Consecuencias Detrimentales de lo Alterno. San Juan: Publicaciones Puertorriqueñas.

Santiago-Negrón, Salvador. (2007). La Violencia en Puerto Rico: Retos de la Salud Pública para la Construcción de la Solidaridad Social. San Juan: Comisión para la Prevención de la Violencia (COPREVI). Recuperado el 11 de agosto de 2007 de http://www.coprevi.org/

Substance Abuse and Mental Health Services Administration (SAMHSA). (2000a). Year-end 1999 emergency department data from the Drug Abuse Warning Network. Rockville, Md.: SAMHSA. 
Substance Abuse and Mental Health Services Administration (2000b). Accreditation Guidelines. Recuperado el 9 de agosto de 2007 de http://www.samhsa.gov/

Substance Abuse and Mental Health Services Administration SAMSHA (2007). Final Revised Guidelines for the Accreditation of Opioid Treatment Programs. Recuperado el 20 de octubre de 2007 de http://www.samhsa.gov/

Travis, Jeremy. (2005). Families and Children. Federal Probation, 69(1), 31-42.

Tomasino, Vincent; Swanson, Arthur J.; Nolan, James \& Shuman, Harry I. (2001). The Key Extended_Entry Program (KEEP): a methadone treatment program for opiate-dependent inmates. Mount Sinai Journal of Medicine, 68(1), 14-20.

Van Wormer, Catherine \& Davis, Diane Rae. (2003). Addiction treatment: A Strength perspective. CA: Brooks-COLE, Thomson Learning Academic Center.

Varas-Díaz, Nelson \& Serrano-García, Irma \& Toro-Alfonso, José (2004). Estigma y Diferencia Social: VIH/SIDA en Puerto Rico. San Juan, P.R.: Ediciones Huracán.

Walmsley, Roy. (2007). World Prison Population List. (6 $6^{\text {th }}$ Ed.). International Centre for Prison Studies. London: Kinas Collage Press.

World Health Organization (WHO). (2004). Neuroscience of Psychoactive Substance Use and Dependence. Geneva: World Health Organization Press.Walmsley, Roy. (2007). World Prison Population List. (6 ${ }^{\text {th }}$ Ed.). London: International Centre for Prison Studies and Kinas Collage Press.

Zarkin, Gary A.; Dunlap, Laura.J.; Hicks, Katherine A. \& Mamo, Daniel. (2005). Benefits and costs of methadone treatment: results from a lifetime simulation model. Health Economics, 14 (11), 1133-1150. 
